\title{
Media archaeology in art practice
}

\author{
Chris Cornish \\ 1a Charnock Road, \\ London E5 8DP \\ chris@chriscornish.co.uk
}

\begin{abstract}
The focus of this paper is the description and contextualisation of series of artworks that I have been developing since 2007, each of which I feel address concepts of what is termed 'media archaeology' - the ability to extract information, objects and environments from either virtual or historical sources and reproduce them in a real world space, in this case the art gallery.
\end{abstract}

Art. Digital technology. Media archaeology. Computer games. Sculpture. Film.

\section{INTRODUCTION}

In 1996 Siegfried Zielinski (1996) defined media archaeology as a 'means to dig out secret paths in history, which might help us find our way into the future'. Since then, Zielinski and other media archaeologists have moved further away from a traditional analysis of archaeology and instead want to illuminate the histories of media software and hardware, giving equal importance to both the discursive formations and material apparatus, the machines and imaginaries, by understanding the boundaries between them as highly permeable and porous (Kluitenberg, 2008).

Widely available computer technology such as camera tracking software, OpenGL extractors and 3D scanning hardware, all allow the measurement, capture and analysis of both real and virtual spaces and objects. These ubiquitous resources create a flux between presumed realities and recorded histories; using these technologies and binding them conceptually to digital reproduction processes, it is possible to physically re-create environments and/or objects which perhaps did once exist and are no more, or were the creation of our imagination to begin with. Umberto Eco (1990) in his book Travels in Hyperreality states that to define history it is crucial to forget and Baudrillard has often argued that medium can create or deny an event or place. With these projects I am aiming to investigate just such boundaries and what happens when geometry and mythology are pulled from one simulation to another.

\section{PRE-FABRICATION / MODULE}

In 2007 I started work on as series of sculptures that would explore the possibilities of extracting geometry from the architecture of computer games and reproduce the elements as a sculptural form. The concept of the crater became the focus for this project - the crater has become a familiar icon in modern history and particularly the documentation of contemporary warfare; a strange, magical interruption through architecture and landscape which bears witness to a lost event. The sculpture represents a meeting point of real and virtual, violence and contemplation.

Utilising a piece of software called OGLE I was able to capture geometry from the computer game Half-Life 2. OGLE is an open source software package developed by Eyebeam OpenLab in the US (Eyebeam, n.d.) that allows for the capture and re-use of 3D geometry data from 3D graphics applications such as computer games, Google Earth and second life. It works much like the standard 'print screen' operation in Windows, yet functions while the user is engaged in a 3D environment. I was quickly able to build up a library of architectural objects (Figure 1) which are used during game play and what soon became apparent was that although the computer game plays like a consistent, seamless environment; it is instead but a thin façade, disguising the complex mechanics of a technical illusion. A game level references the real world both physically and architecturally, yet it is a place without history or narrative; like a theatre set, after each game all that has happened is deleted and reset. Architecture does not age, the weather does not change, instead the same sequences repeat, simulations run and tests complete. It is interesting to think of an environment where multiple time lines co-exist, but where all possibilities have been tested and prepared. For every architectural element, there exists a number of alternative versions held in a memory library, ready to be seamlessly swapped. Architecture becomes predetermined and dislocated from time. 


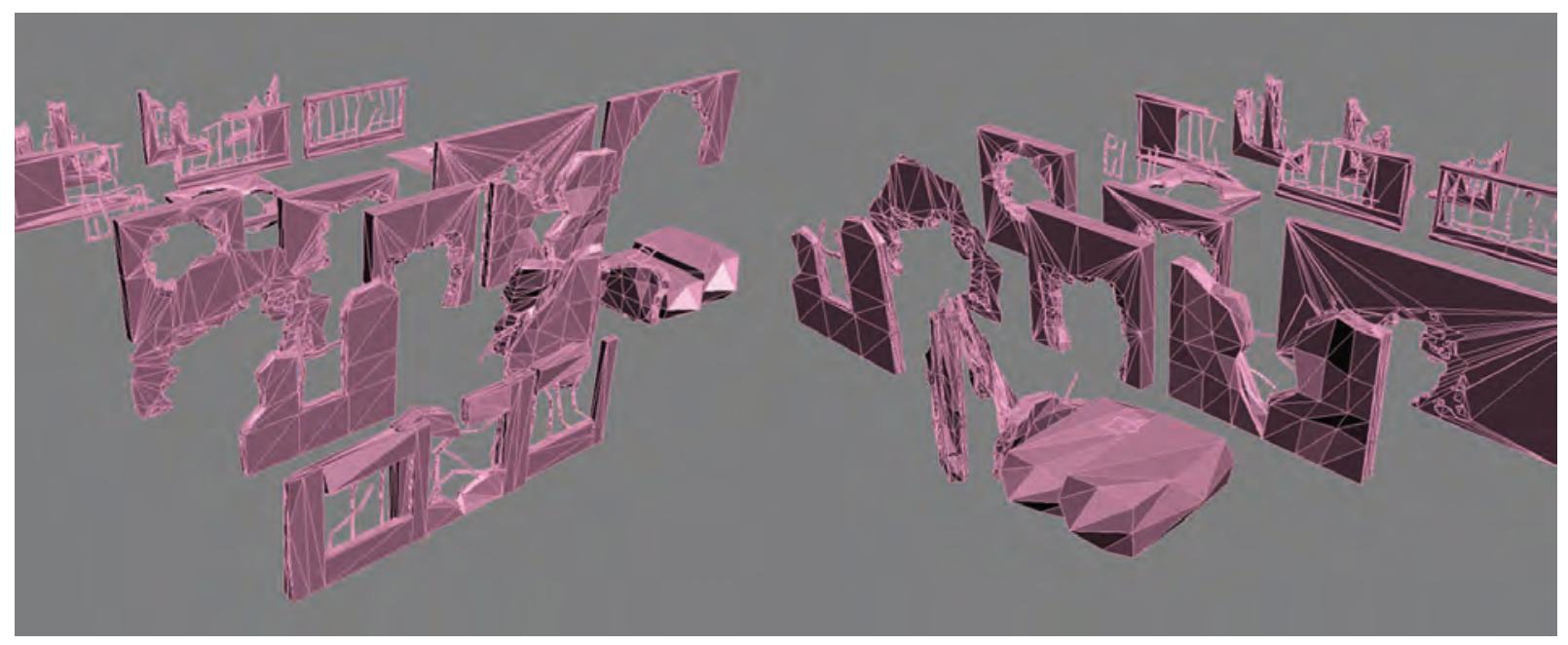

Figure 1: Geometry captured from the computer game Half-Life 2

The idea of pre-designed destruction is very poignant to me. The concept that an environment could shift between modes of representation in response to the presence of a single viewer seems to confirm that history in this situation was indeed porous and open to manipulation. For the sculpture pre-fabrication, crater 2008 I reproduced a section of this modular architecture, a unit that would be used to denote damage, a crater (Figure 2). In creating the object in the real world, I was attempting to test and challenge its original purpose - the crater, once a random representation of violence and power becomes an inert object; the result of a reductive process of architectural design. It also becomes dislocated from its original representational mode, the illusionary and permeable space of the computer game is replaced by the hard white walls of the gallery; the object becomes heavy, stuck; it obtains a strange mass.

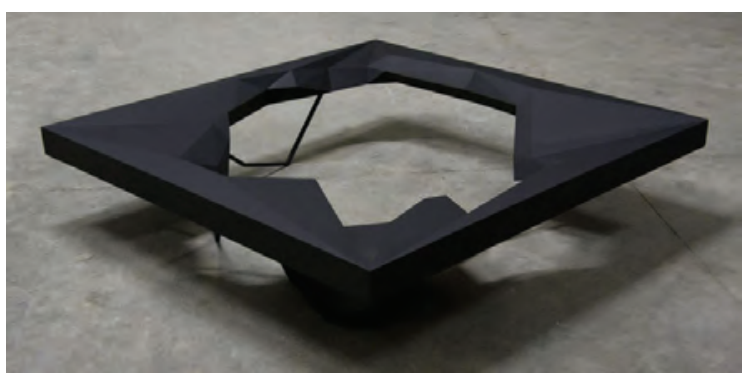

Figure 2: pre-fabrication, crater, 2008, mixed media, $150 \mathrm{~cm} \times 150 \mathrm{~cm} \times 35 \mathrm{~cm}$

Notions of distillation, modularity and transition are explored further in the series of sculptures during 2007 - 2008. In the work module 02, transporter
2007-2008 (Figure 3) the 3D geometry of a crater is collapsed into a layered form, the structure and shape of the object shifting due to the matt surface finish. The object mimics the screen based representation of computer games, however traditional perspective cues such as shading, occlusion and foreshortening are removed. The spacial rules become scrambled in the transition between the virtual and real world.

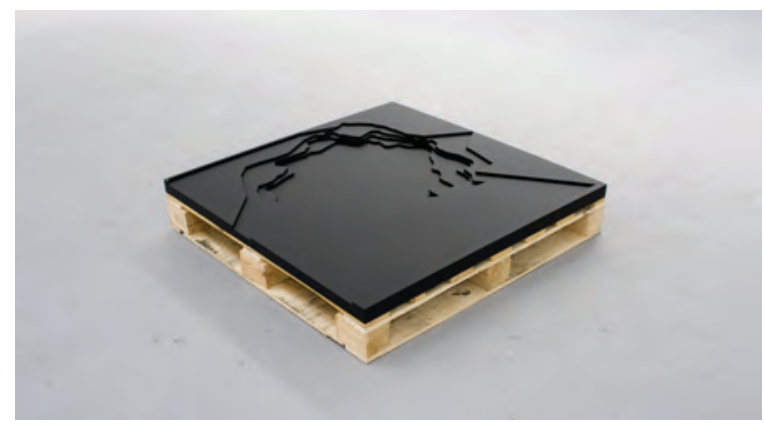

Figure 3: module 02, transporter, 2007-2008, wood, paint, $100 \mathrm{~cm} \times 100 \mathrm{~cm} \times 20 \mathrm{~cm}$

It is this idea of transition I explore in the work module 07, portal 2008 (Figure 4). Chroma key paint (normally used in visual effects to aid the erasure of the subjects' surroundings) is used to create a bridge between different versions of reality, the sculpture situated in neither. This again references computer games and the use of a 'portal' as a vehicle of transcendence upon the start and finish of a level and the death of a player. 


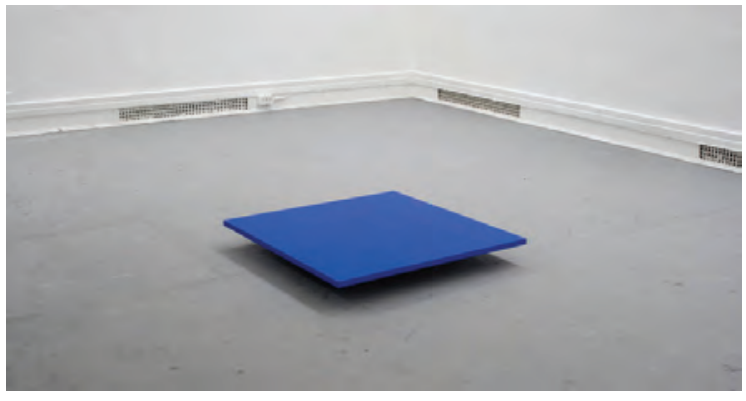

Figure 4: module 07, portal, 2008, wood, chroma key paint, $100 \mathrm{~cm} \times 100 \mathrm{~cm} \times 20 \mathrm{~cm}$

\section{THE ZONE}

In 2009 I began a body of research which furthered my exploration of the boundaries of real and virtual and also the relationship between the mechanics of digital technology and the actualisation of objects from what could be considered an archaeological source.

The term 'digital archaeology' is generally linked to the process of collecting 3D data from existing objects deemed to have historical worth and their re-presentation in a virtual environment to aid learning and dissemination. This action relates well to the traditional analysis of archaeology mentioned in my introduction; the systematic description of a discourse object. Instead I wanted to find a way to utilise these same digital technologies to explore and recover histories that exist on the margins of reality and mythology, what Zielinski called 'secret paths in history'.
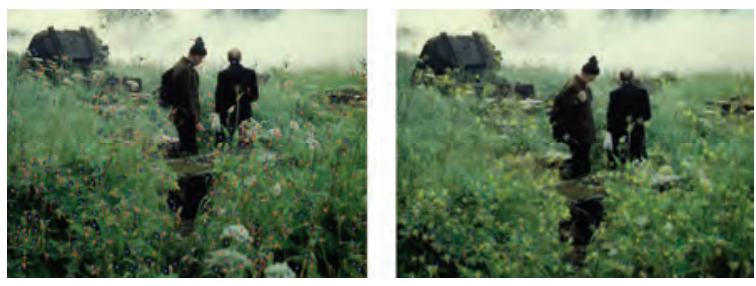

Figure 5: Still images from Stalker with tracking markers

I chose the 1979 film Stalker directed by Andrei Tarkovsky (1979) as the subject of my research. Stalker is a science fiction film which depicts the protagonist's journey into a landscape known as the 'Zone' which has the supposed potential to fulfil a person's innermost desires. Residual effects of an unnamed previous occurrence have transformed an otherwise mundane rural landscape scattered with ruined buildings into an area where the normal laws of physics no longer apply (Wikipedia, n.d.). The 'Zone' is described as a place of extreme, but unseen danger, the lead character, the Stalker, navigating by throwing ahead nuts and bolts tied to cloth to test the route before proceeding.

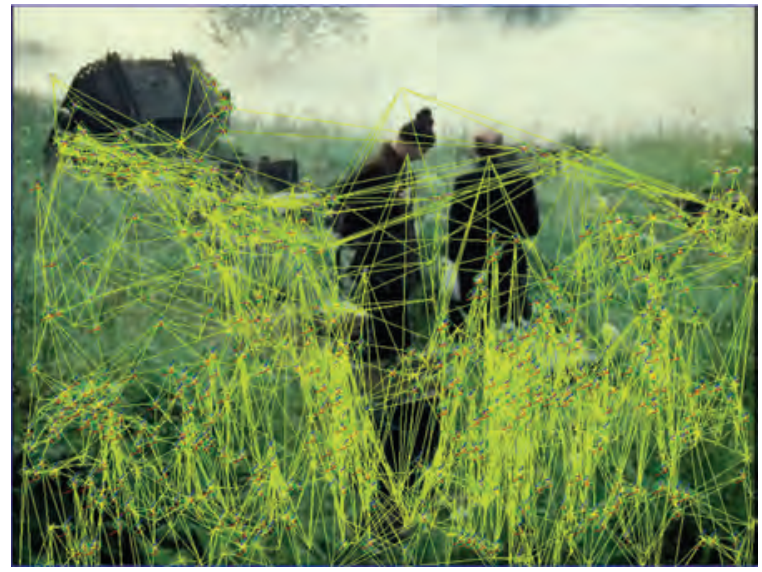

Figure 6: Still images from Stalker with wireframe mesh

As well as the mythology on film, the area of the 'Zone' developed its own real world mythology; it predicted the nature of the landscape that enveloped the ruins of Pripyat after the Chenobyl disaster in 1986 and also is thought to have caused the untimely deaths of many people involved in the production of the film, later attributed to toxins present at the shooting locations.

For a series of sculptures I wanted to extract and reproduce real scale sections of landscape from the Zone, using digital technology to realise a landscape which is infamously difficult to navigate, record and experience; an environment that sits on the boundaries of multiple histories.

I analysed sequences from the film for feature details and parallax movement using camera tracking software (Figure 5), a tool used in the visual effects world to recover camera movement so that computer generated characters and objects can be placed in filmed environments. The software identifies points of interest and tracks their movement for the duration of the clip; tracking markers near the camera will move more than those further away, allowing the software to deduce a cloud of 3D points in space.

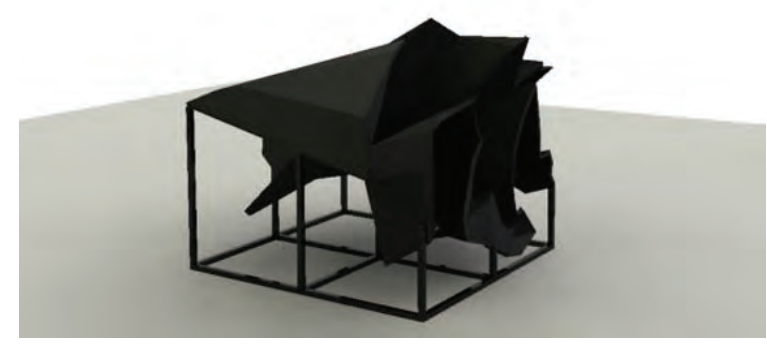

Figure 7: Computer generated prototype for the zone project 
The 3D point cloud is joined to form a wireframe 3D mesh (Figure 6). This 3D shape is a simplified but accurate representation of the environment featured in the film clip.

The virtual shape is then cut into meter square sections and manufactured at a real world scale from aluminium composite and installed in the gallery (Figures 7 and 8 ).

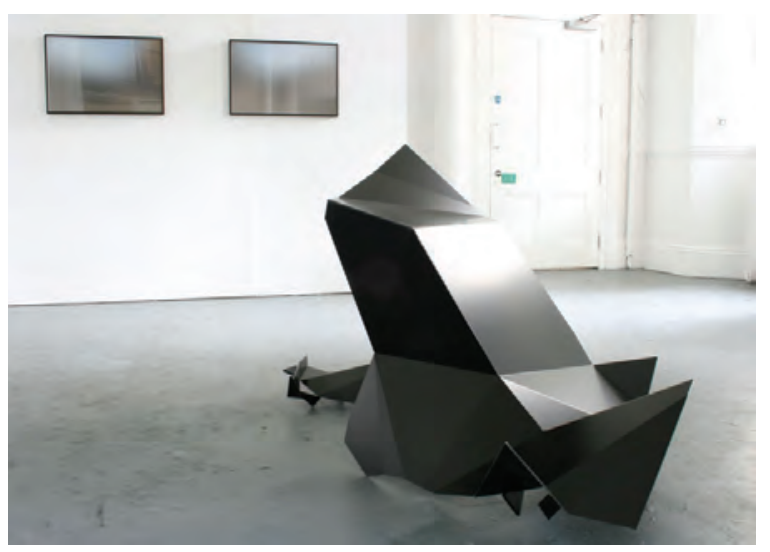

Figure 8: the zone, 2008-2009, aluminium composite, acrylic, $100 \mathrm{~cm} \times 100 \mathrm{~cm} \times 60 \mathrm{~cm}$

In the manifestation of the sculptural objects I was keen to avoid a perfect replication of the original section of landscape and instead wanted to allow the technology to impact on its representation. While the points in space remain faithful to the original landscape, the way the software joins the dots is not faithful to the landscape and is instead dependent on the view through the camera. This creates a unique, abstracted landscape whose geometry is formed by the viewer's own presence. Just as the original landscape responds to its inhabitants, I wanted the new landscape to develop a narrative and structure of its own, exposing the technology intrinsic to its creation. There are also some interesting parallels between the technology I was using to create the work and actions within the film. For example, my technique of surveying the scene is not too different from the Stalker's use of throwing nuts and bolts to navigate - both plotting random points in space to locate and maintain physicality.

\section{SAMPLE AND HOLD}

The concept of random points and the interpretation of an environment through technology became the starting point for a project which I am currently working on at the time of writing this paper. Titled Sample and Hold 2010 the project is an attempt to capture what could be considered the aura given off by the interaction of architecture as interpreted through the singular perspective of a laser scanner. Again this could be considered a form of archaeology. However, it is documenting the tools and technology in conjunction with the real world object. The results produced are specific to the software and hardware apparatus used to record and process them (Figure 9).

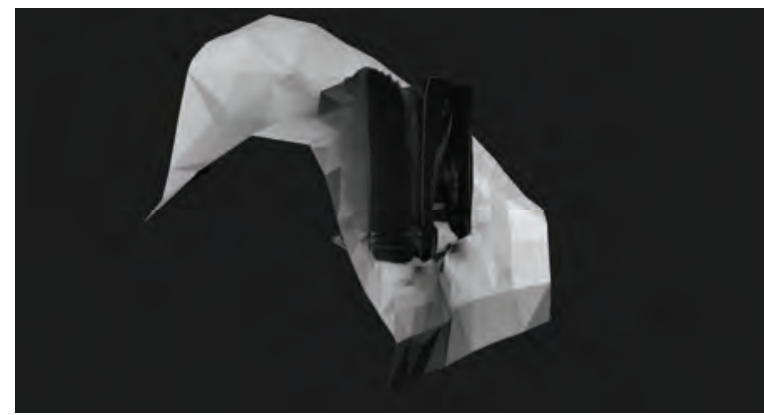

Figure 9: Sample and Hold, initial test using Poisson Surface Reconstruction

Machines and apparatus have influenced the production of art work throughout history. In 1435 Leon Battista Alberti published what is considered the first treatise on the laws of perspective, Della pittura, included was a description of a drawing device which Albercht Dürer later developed in his illustrated books Underweysung der Messung, 1525 (figure 10). Known as a drawing frame or perspective machine, it consisted of a square wooden frame through which a number of horizontal and vertical lines are threaded to produce a grid, offset towards the viewer was a vertical rod whose height matched that of the centre of the grid. The viewer placed their eye next to the tip of the rod and looked through the wooden frame to the scene beyond, seeing a representation of space that was divided into small sections and could then be easily transferred to paper prepared with the same grid sectioning (National Portrait Gallery, 2009). Over time as this concept was developed and modified, the different machines were with a common theme - Alberti's grid, Alberti's window, the draughtsman's net the artist's veil. All refer to the idea of entrapment, the idea that a space can be somehow teased into a $2 \mathrm{D}$ plane, extracted from the real world and contained within a system of mathematical laws. The single point perspective system in this case creates a world order within the image that is separate from the physical reality of its origin, which has been removed and displaced (Naughton, n.d.). 


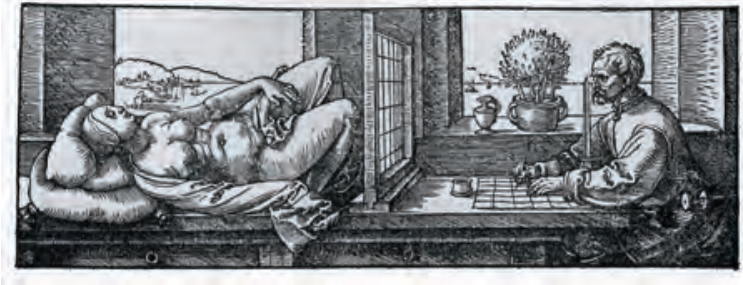

Figure 10: illustration of a perspective machine published by Albrecht Dürer in The Painter's Manual in 1525

A direct connection can be made between perspective machines and contemporary techniques for capturing 3D information from real world objects and recreating them in computer space - in particular 3D scanners. A large area 3D scanner (a device that can 3D scan and produce accurate digital representations of large spaces) was used to scan a room in the Woburn Research Centre, part of the Slade School of Fine Art, and a building that used to house the Courtauld collection. The scanner used was a FARO Photon 120 , which fires out 976,000 points per second and records a 3D co-ordinate for each point which is accurate to plus-minus $2 \mathrm{~mm}$ for a distance up to $120 \mathrm{~m}$. During the surveying process the data is filtered and processed so that that which is considered inaccurate is discarded. It is precisely this data which I am interested in: the 'noise' usually produced when the laser point hits complex geometries and shiny surfaces. This collection of noise data is then used to reconstruct surfaces using an algorithm known as Poisson Surface Reconstruction (9) - a method which fits a 3D surface to a point set and which is very robust in relation to noise and lack of uniformity. The resulting surfaces are both abstract and nonrepresentative but perversely also a legitimate depiction of the environment as filtered through contemporary hardware and software: apparatus that let us experience architecture and material beyond that which the naked eye can perceive.

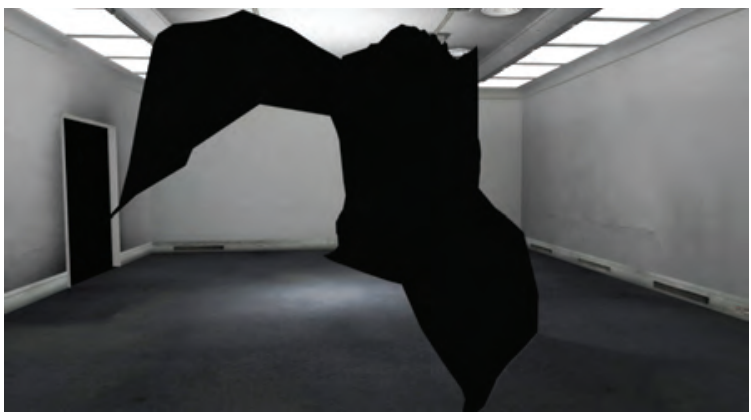

Figure 11: Sample and Hold, computer generated test of installation view

Although still a work in progress, the aim of this project is to manufacture the surfaces produced and install them in the original environment in which the data was collected. The finished sculptures become a very tangible link between the real, the virtual and the tools that were intrinsic to the realisation process (Figures 11 and 12), a contemporary archaeological site.

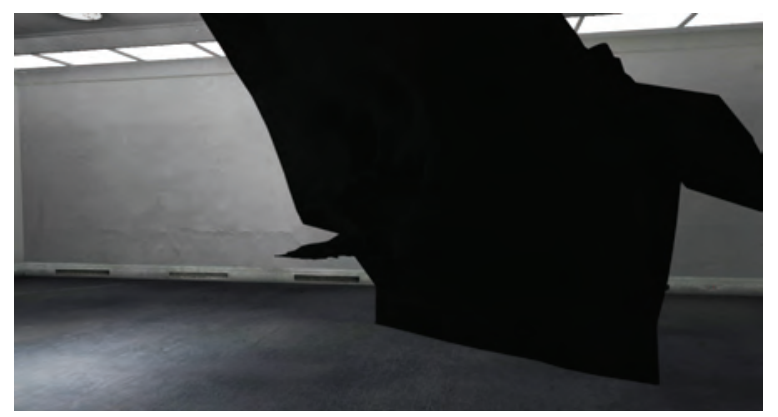

Figure 12: Sample and Hold, computer generated test of installation view

\section{CONCLUSION}

Through the description of these art works I hope to have demonstrated that contemporary technology can be used to explore environments and narratives that were otherwise closed or forgotten. A supplementary history exists within this relationship of tool and resource. This flickers between the boundaries of what is considered real and virtual, and is becoming more relevant and more valid. As technology advances, history and therefore archaeology becomes non-linear, and can be re-read, re-presented and re-imagined.

\section{REFERENCES}

Eco, U. (1990) Travels in Hyperreality, Harvest Books, US.

Eyebeam (n.d.) Ogle: the OpenGLExtractor. http://ogle.eyebeamresearch.org

Kazhdan, M, Bolitho, M and Hoppe, H. (2006) Poisson Surface Reconstruction. In Symposium on Geometry Processing 2006. Cagliari, Sardinia, Italy, 26-28 June, pp. 61-70. Eurographics Association, Aire-la-Ville, Switzerland. http://www.cs.jhu.edu/ misha/MyPapers/SGP06.pd f

Kluitenberg, E. (2008) Delusive Space: Essays on Culture, Media and Technology, Institute of Network Cultures Nai Publishers, Rotterdam.

National Portrait Gallery, (2009) The Drawing Machine.

http://www.npg.org.uk/learning/digital/portraiture/pe rspective-seeing-where-you-stand/the-drawingmachine.php 
Naughton, R (n.d.) Adventures in Cybersound. http://www.acmi.net.au/AIC/DRAWING MACHINE S.html

Tarkovsky, A. (1979) Stalker. Mosfilm, Russia
Wikipedia.(n.d.) Stalker (film).

http://en.wikipedia.org/wiki/Stalker (film) (28 March 2010)

Zielinski, S. (1996) Media Archaeology. http://www.ctheory.net 